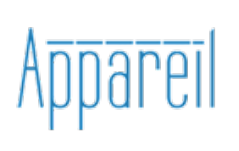

Appareil

14 | 2014

Esthétiques latino-américaines : penser à rebours

\title{
Mexique : le défi esthétique de la disparition forcée
}

Jean-Louis Déotte

\section{(2) OpenEdition}

Journals

Édition électronique

URL : http://journals.openedition.org/appareil/2092

DOI : 10.4000/appareil.2092

ISSN : 2101-0714

Éditeur

MSH Paris Nord

Référence électronique

Jean-Louis Déotte, «Mexique : le défi esthétique de la disparition forcée », Appareil [En ligne], 14 |

2014, mis en ligne le 05 décembre 2014, consulté le 30 juillet 2020. URL : http://

journals.openedition.org/appareil/2092 ; DOI : https://doi.org/10.4000/appareil.2092

Ce document a été généré automatiquement le 30 juillet 2020.

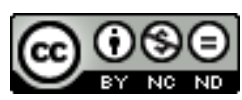

Appareil est mis à disposition selon les termes de la Licence Creative Commons Attribution - Pas d'Utilisation Commerciale - Pas de Modification 4.0 International. 


\title{
Mexique : le défi esthétique de la disparition forcée
}

\author{
Jean-Louis Déotte
}

1 Le Mexique qui, à la différence de la plupart des États latino-américains, avait échappé aux coups d'État militaires, et à une politique terroriste programmée de disparition, découvre le mot "disparition forcée " ${ }^{3}$. Certes la répression sanglante du 2 octobre 1968 sur la Place des Trois Cultures, puis la lutte contre l'extrême-gauche au début des années 1970 , entraînèrent la disparition de plusieurs centaines de personnes ${ }^{4}$. 


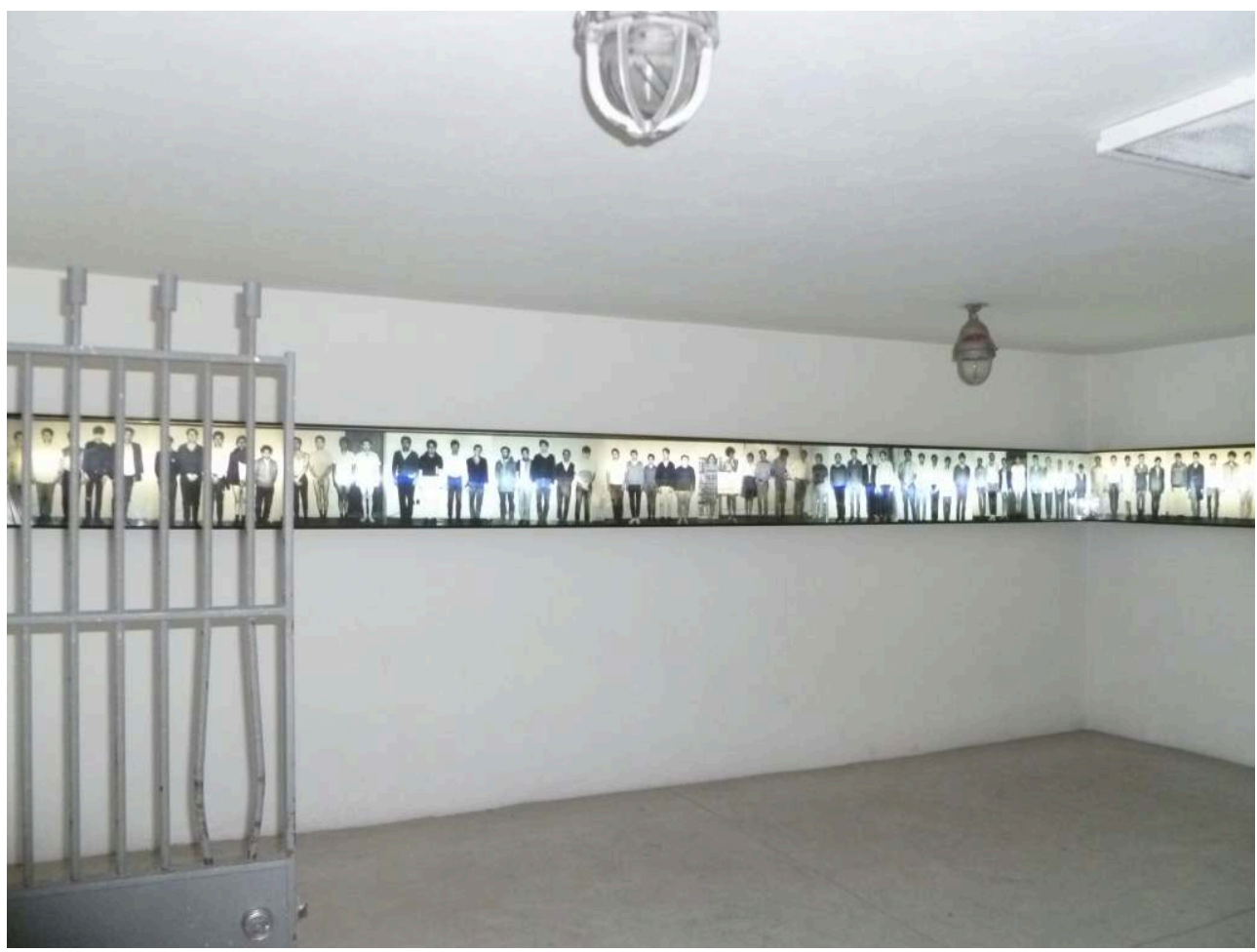

Centre culturel de I'UNAM, Tlatelolco

2 Mais désormais le Mexique doit affronter l'« événement » de la pratique terroriste de la disparition en le conceptualisant et en l'esthétisant. Il doit accepter, à son corps défendant, l'idée que l'hyper-violence qui règne dans certaines régions, en particulier dans les États frontaliers du Nord, dans l'impunité la plus totale, et qui avait constitué la matière de l'écrivain chilien Roberto Bolaño $\left(2666^{5}\right)$, constitue sa réalité sociopolitique, malgré son aspiration à une modernité radicale. Le paradoxe du Mexique contemporain se résume à la formule foucaldienne: comment la bio-politique revendiquée ( faire vivre, laisser mourir ») peut-elle se concilier avec le « laisser vivre, faire mourir » archaïque $^{6}$ ?

C'est peut-être la fantasmagorie du métissage, par ailleurs largement alimentée par les études de l'excellent $\mathrm{S}$. Gruzinski' ${ }^{7}$, qui vole en éclat. Car même si le « système » colonial « des castes ${ }^{8}$ a été officiellement abandonné depuis l'Indépendance et la Réforme, il n'en reste pas moins que la couleur de peau d'un individu reste un facteur implicite de classement social. Ce n'est pas un hasard si le premier discours de l'actuel président mexicain à l'ONU a porté sur les discriminations dont les Indiens de son pays étaient les victimes, ni si la loi de l'État fédéral exige qu'à l'entrée des bars et restaurants on trouve en bonne place cette affiche : Se obliga a que todo establecimiento exhiba en un lugar visible el horario de funcionamiento, así como un letrero con la leyenda siguiente: "En este establecimiento no se discrimina por motivos de raza, religión, orientación sexual, condición física o socioeconómica ni por ningún otro motivo". También se deberá anunciar un número telefónico para quejas y emergencias, así como la dirección de la Procuraduría General de Justicia del Distrito Federal. 2009. ${ }^{9}$

4 Par ailleurs, l'actuel Président a promis en 2013 qu'au niveau fédéral, la disparition forcée rentrerait dans le cas du Code pénal, ce qui n'était pas le cas auparavant ! 
5 Cette modernité mexicaine réaffirme que nul n'est exclu du cercle de l'humanité. Cette volonté d'inclusion, qui a son origine dans le mouvement européen des Lumières, et en particulier dans la reconnaissance de l'humanité de la folie ${ }^{10}$, contredit clairement la thèse de Foucault d'une exclusion générale des « handicapés du signe » par l'invention de l'enfermement dans l'Hôpital général à l'époque "classique " ${ }^{11}$. Le problème philosophique des foucaldiens est de confondre l'ordre du fait et l'ordre du droit, ce qui est inévitable dans le cadre d'une stricte philosophie du pouvoir comme unique substance.

6 Le Mexique où la pratique mafieuse du rapt était fréquente ${ }^{12}$, est mis à l'épreuve d'un événement politique : la disparition forcée d'un groupe de jeunes gens, élèves d'une école normale rurale, issus de milieux pauvres, d'origine amérindienne, réputés pour leur activisme politique dans un de ces États, comme celui d'Oaxaca, où la lutte entre les enseignants et les autorités peut prendre des formes très violentes (Oaxaca: 2006). Peu à peu, on prend conscience de la difficulté à circonscrire cet événement, d'où la référence immédiate au slogan des Mères argentines de la Place de Mai (« réapparition en vie!») et l'utilisation des photos des disparus comme armes politiques. Il nous faudra dès lors reconsidérer le rôle de la photographie, non pas comme art, mais dans la constitution technique des groupes socio-politiques.

7 Là où la fête des Morts s'impose depuis toujours comme le grand moment festif de l'année (bien que concurrencée de plus en plus par Halloween et ses sorcières), il est difficile aux artistes « engagés » de rendre hommage aux disparus. Dans la rue centrale d'Oaxaca, libérée momentanément des tentes des enseignants contestataires (car le mouvement perdure en 2014), où s'exposent selon la tradition, par dizaines des autels des morts, le sable répandu permet à la fois de supporter une immense tête de mort, mais aussi quarante-trois autres de petites dimensions, comme si les disparus n'étaient pas considérés comme tels mais plutôt comme des individus dont le décès serait avéré. Il convient alors de les honorer comme n'importe quel défunt. Ce qui revient à accepter les allégations de l'État dans une ambiance joyeuse où le militant danse au rythme d'une samba! Autour, défilent des groupes de "Catrina », de squelettes «féminins » recouverts de tenues européennes sexy.

8 Certains pensent que les images sont comme les objets techniques: neutres. À disposition. Que ce serait l'utilisateur, comme le spectateur d'une œuvre d'art, qui en ferait un bon usage ou non, esthétique ou politique. L'analogie peut s'avérer riche, à condition de rappeler qu'un outil n'est pas neutre. Encore moins un ensemble d'outils appelé machine. Parce qu'une machine transforme le milieu naturel auquel elle s'applique et le transforme en « milieu associé » en intégrant un certain nombre de ses structures $^{13}$. Il y a belle lurette que les images ont intégré les structures de tel ou tel milieu socio-historique, le modifiant en retour. Ce qui implique qu'un corpus d'images doit être mis en rapport avec son milieu associé, en l'occurrence, social et politique, qui lui donne sens. Le livre ${ }^{14}$ de photographies de l'Algérie de la guerre civile d'Omar Daoud est de ceux-là : il doit être compulsé en rapport avec le collectif algérien des « mères de disparus» qui lui donne sens. Mais ce collectif n'aurait pas existé sans ces représentations, sans ces témoignages, sans ces lettres envoyées en vain par des parents de disparus politiques aux représentants d'un État qui n'a pas besoin de peuple. Le peuple algérien pour l'État du même nom est un peuple en trop: les revenus de l'exploitation de l'énergie pétrolière constituant une rente largement suffisante. Mais 
tous les peuples d'États dont les ressources sont de cette nature ne sont-ils pas réduits à cette situation, la Russie de Poutine faisant partie du lot?

Illustrations 2. Omar Daoud : groupe de disparus

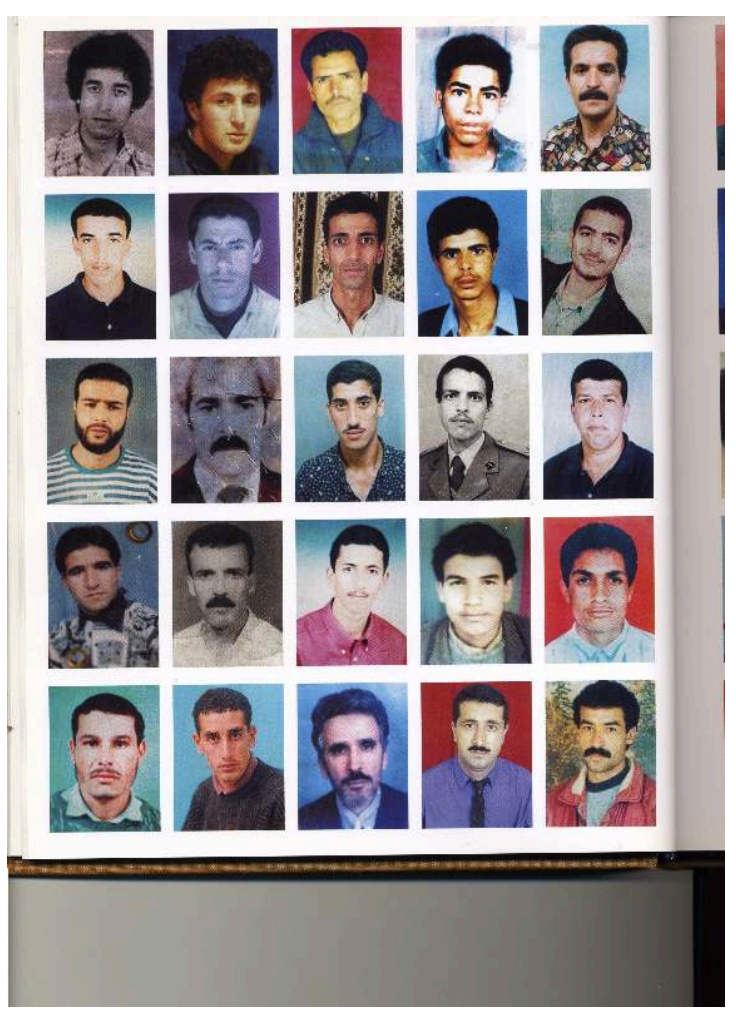

Ces images politiques nous les retiendront particulièrement, parce qu'elles sont à l'entrecroisement de deux logiques indépendantes au moins : la politique, la technique, ici imageante. Ces deux logiques elles-mêmes dépendent de régimes différents du symbolique, de la cosmétique. On peut caractériser rapidement les régimes du symbolique et donc de la mémoire collective: le régime narratif qui est le fait des sociétés contre l'État et des sociétés traditionnelles ou païennes, le régime de la révélation qui est le fait des sociétés théologico-politiques comme le christianisme ou l'islam, le régime délibératif pour les sociétés démocratiques-capitalistes. Ce sont ces régimes de la cosmétique qui permettent aux groupes sociaux de s'individuer.

Chaque régime entretient un rapport spécifique à l'événement: le premier fait tout pour l'exténuer au nom de la répétition du Même (l'ordre social légué par les ancêtres mythiques), le second le transforme en avènement majeur (cf. l'écriture des tables de la Loi par Moïse) conditionnant tout espoir de retour, le troisième s'ouvre au grand large de l'indétermination symbolique. Si l'on suit cette tripartition, le monde pré-colombien et ses empires incas, aztèques, etc., s'introduit en coin entre les deux premiers rapports, comme si le monde de la répétition mythique du Même était entré dans un devenir impérial donnant ainsi une place spéciale aux envahisseurs européens ${ }^{15}$.

En effet, Lefort à la suite de sa lecture de Tocqueville ${ }^{16}$ détermine la démocratie comme régime social-historique par l'indétermination croissante des repères symboliques, sur les ruines d'une société théologico-politique de l'incorporation et de l'incarnation (cf. l'Ancien Régime en France). La thèse est la suivante : à partir du moment où la société ne s'incarne plus dans le corps du roi, tout ce qui apparaissait jusqu'alors comme donné 
d'avance par une sorte de don divin du sens ou de destination (la finalité de l'ordre social, les partages sociaux et sexuels, les moyens de parvenir aux buts collectifs, etc.) devient objet d'une délibération et donc d'un débat où les parties ne cessent de se diviser sur tout et n'importe quoi.

Non seulement pouvoir, savoir et loi se désintriquent, mais cette première différenciation en entraîne d'autres; on peut parler d'ontologies régionales, puisque des pans entiers de réalité se détachent des autres, acquerrant à chaque fois légitimité, savoir et pouvoir (la religion, la science, l'éthique, l'esthétique, la technique, etc.). C'est le constat que faisait déjà Schiller ${ }^{17}$ à l'extrême fin du XvIII ${ }^{\mathrm{e}}$ siècle, celui d'une humanité et d'un homme divisés. De là selon lui et à partir de lui, l'utopie d'un homme et d'une société réconciliés du fait de l'esthétique. Il est évident que ce mouvement de différenciation démocratique s'entretient de lui-même et que les limites du symbolique reculent constamment, générant selon Lefort une angoisse de démembrement de ce qui restait de corps social. Les fictions catastrophistes d'une société unidimensionnelle, d'un spectacle généralisé aliénant tout comportement authentique parce que supposé harmonieux, les idylles rousseauistes d'une bonne société ne connaissant pas l'inégalité, etc., sont à la mesure de cette différenciation croissante.

13 Très tôt, Lefort a analysé le totalitarisme nécessairement moderne, comme une tentative pour générer fantasmatiquement un corps social et politique réunifié sous la tutelle d'un égocrate (Hitler, Staline, Mao, etc.). Le totalitarisme était donc décrit comme le risque majeur de la démocratie, un désir de fusion logé au cœur de l'expérience démocratique de la division.

14 Cet horizon délibératif est au cœur de la conception par Rancière de l'action politique. En effet, ceux qui n'avaient pas encore de places socialement reconnues, les sans-part, sont amenés, sur un fond d'égalité essentielle entre les locuteurs humains, à constituer un objet collectif de litige, à configurer une place publique et donc à argumenter face à ceux qui, a priori, ne les voyaient même pas (ceux qui prennent part à l'ordre social, la polis). Bref, d'invisibles, ils doivent partager autrement le sensible pour se rendre visibles. Le recours aux images, comme à la littérature, devient essentiel. Les images sont alors des armes politiques agissant même inconsciemment puisque c'est l'ensemble de notre sensibilité qui au bout du compte accepte de voir ce qui jusqu'alors restait dans l'ombre. Un nouveau milieu associé surgit donc de ce partage du sensible. Et comme la politique est affaire d'apparences que l'on donne de soi-même (W. Benjamin), toujours à son insu (Arendt), les agissants sur la scène publique doivent être appareillés pour exister aux yeux les uns des autres. Ils doivent apparaître au mieux d'eux-mêmes (c'est toujours une affaire de parure et d'éloquence) se soumettant à l'appareil esthétique dominant qui fait le tri entre ce qui peut surgir comme événement et ce qui reste inconcevable. On se souvient des portraits de groupes de Communards à Paris en 1871, tous fiers et armés sur leurs barricades, défiant l'ennemi versaillais qui plus tard se saisira de ces photos pour les condamner sur place et les fusiller. La plupart disparurent sans restes dans des fosses communes. La photographie constitue alors le seul témoignage de leurs existences. En même temps, la photographie fut utilisée comme arme politique par les vainqueurs eux-mêmes pour justifier la répression de la Semaine sanglante : la fusillade des «otages de la rue Haxo » fut reconstituée après coup comme une scène de théâtre, avec des acteurs, et les photos largement diffusées dans la presse de l'État dirigé par Thiers. 
Mais chez les uns et les autres (de Lefort à Rancière, etc.), c'est le schéma marxien de la lutte des classes qui continue de s'imposer. Au fond, la division est essentiellement sociale et politique. Avec Lyotard, le champ potentiel de la division s'élargit aux conflits entre régimes du symbolique ${ }^{18}$. C'est que Lyotard, qui avait participé activement au mouvement de libération de l'Algérie ${ }^{19}$, fit très tôt l'analyse de la situation coloniale, où une norme s'impose à une autre, la laissant sans voix, par exemple la norme délibérative s'imposant à la norme de révélation. Les conflits peuvent concerner des régimes hétéronomes de l'image, voire des conflits sur le refus de l'image: Lyotard mènera à partir de Kant toute une réflexion sur le sublime comme «présentation qu'il $\mathrm{y}$ a de l'imprésentable ». On assiste donc à un approfondissement de l'indétermination symbolique quand les repères culturels les plus intégrés implicitement s'exacerbent dans la confrontation avec d'autres repères culturels. Il y a donc des différends cosmétiques.

Du politique au social, du social au culturel, une révolution était en marche, souvent à grand bruit. Mais les événements les plus conséquents sont souvent silencieux : la remise en cause du partage social des sexes, le féminisme, la question du genre, la transexualité, la revendication d'une non-discrimination concernant les homosexualités, ce mouvement qui touchait à la division entre sexes du fait de leur récente indistinction pouvait être encore qualifié de démocratique. Mais l'homoparentalité ? Mais les mères porteuses? Mais les greffes d'organes? Ce sont alors les repères anthropologiques qui à leur tour firent question, du fait de leur remise en cause, provoquée par des avancées sociales et techniques considérables. Ce qui vient en retour saper la symbolicité et la cosmétique. D’où, dans le cadre de la bio-politique la lutte de l'État mexicain contre toutes les discriminations.

17 À cela s'est ajoutée la remise en cause d'un partage symbolique essentiel à l'humanité : celui entre les morts et les vivants du fait de la politique terroriste d'État inaugurée par les nazis (politique NN : Nuit et Brouillard). Cette politique est distincte des génocides, car elle vise des individualités. Plus l'individuation dans une société est forte, plus les singularités entretiennent entre elles de nombreuses relations, plus les agissants sont des apparaissants, plus la tentation est grande pour un État terroriste d'affaiblir les réseaux interpersonnels sans lesquels il n'y a pas de véritable individuation. En effet, si un groupe n'était qu'une somme d'individus déjà constitués, en faire disparaître un, serait sans grand effet. Le groupe trouverait même des raisons supplémentaires de lutter, par esprit de vengeance. Pour s'attaquer au réseau en tant qu'il est constitutif des individus, il faut plus efficacement s'attaquer au processus psycho-social par lequel les individus vont vers davantage d'individuation. C'est Simondon ${ }^{20}$ qui permet de comprendre qu'un individu de groupe est bien davantage qu'un groupe d'individus.

Pour le dire autrement, la guerre classique met en cause des groupes d'individus, où les individus ont entre eux des relations déjà établies et stables, la plupart du temps hiérarchiques (l'armée), la "sale guerre » de l'Algérie au Mexique d'aujourd'hui, des individus de groupe où le groupe est en cours d'individuation, où les individus s'ouvrent toujours plus à de nouvelles relations entre eux. La " sale guerre " génère de l'angoisse chez chacun parce qu'elle s'attaque au transindividuel, au processus par lequel des individus en relation s'individuent en rapport à l'individuation du groupe qui les porte et qu'ils portent. C'est alors le pré-individuel qui envahit tout.

19 La guerre classique troue les réseaux relationnels déjà constitués, les individus survivants après un travail de deuil doivent contourner les vides laissés par les pertes 
humaines. La nouvelle guerre, qui est essentiellement une guerre civile, une guerre menée par l'État terroriste et ses gangs contre la société, fait régresser le processus d'individuation. Elle est, à proprement parler, antisociale. C'est la raison pour laquelle, l'angoisse, qui est un retour du préindividuel, submerge les individus. L'angoisse provoquée par la disparition forcée d'un proche est beaucoup plus invalidante que sa mort avérée, suivie des rituels funéraires, lesquels replacent le décédé dans une généalogie. La mort prolonge, d'une certaine manière, les rapports que les vivants entretenaient entre eux, tout en instaurant entre le mort et eux une césure qui va devenir de plus en plus grande (cf. la Fête des morts le 2 novembre au Mexique). La disparition, au contraire, c'est brusquement l'absence de liens: l'individuation s'effondre. D'autant que les individus qu'on a fait disparaître l'ont été comme apparaissants au mieux d'eux-mêmes.

Plus radicalement, si un groupe s'individue du fait de l'individuation de ses membres, certains d'entre eux émergent comme étant des sur-individus (des «leaders») : des individus reconnus par tous comme des représentants indiscutables (politiquement, artistiquement, socialement, etc.). Les atteindre, en les faisant disparaître, c'est en quelque sorte priver le processus d'individuation de pilotes. La "sale guerre " est sélective, elle engloutit le fils aîné et non pas le cadet, le leader de l'organisation communautaire et non pas le militant lambda, le chanteur étoile et non pas un fan. Le nombre de disparus au Chili sous Pinochet fut limité, ce qui n'entrava pas la politique de terreur, bien au contraire. Les purges de Staline, surtout dans les années 1930, furent davantage " chirurgicales ", même à très grande échelle, que génocidaires. Alors que le nazisme était essentialiste (principe de la race) et donc génocidaire.

C'est la raison pour laquelle les photos d'Omar Daoud sont à la fois psychiques et collectives, ne se contentant pas, comme le ferait un Boltanski, de livrer des photos d'identité agrandies et pour cela floues, d'anonymes. Les disparus sont là, mais aussi le milieu géographique, rural ou urbain; social : les lieux conviviaux, le café, la rue, les places; le travail : le portage, la cuisine, la lessive; la maison: le milieu familial, la mère, la chambre à coucher, la cuisine, l'enfance, le passé, la phratrie. 
Illustration 3. Photos des disparus mexicains

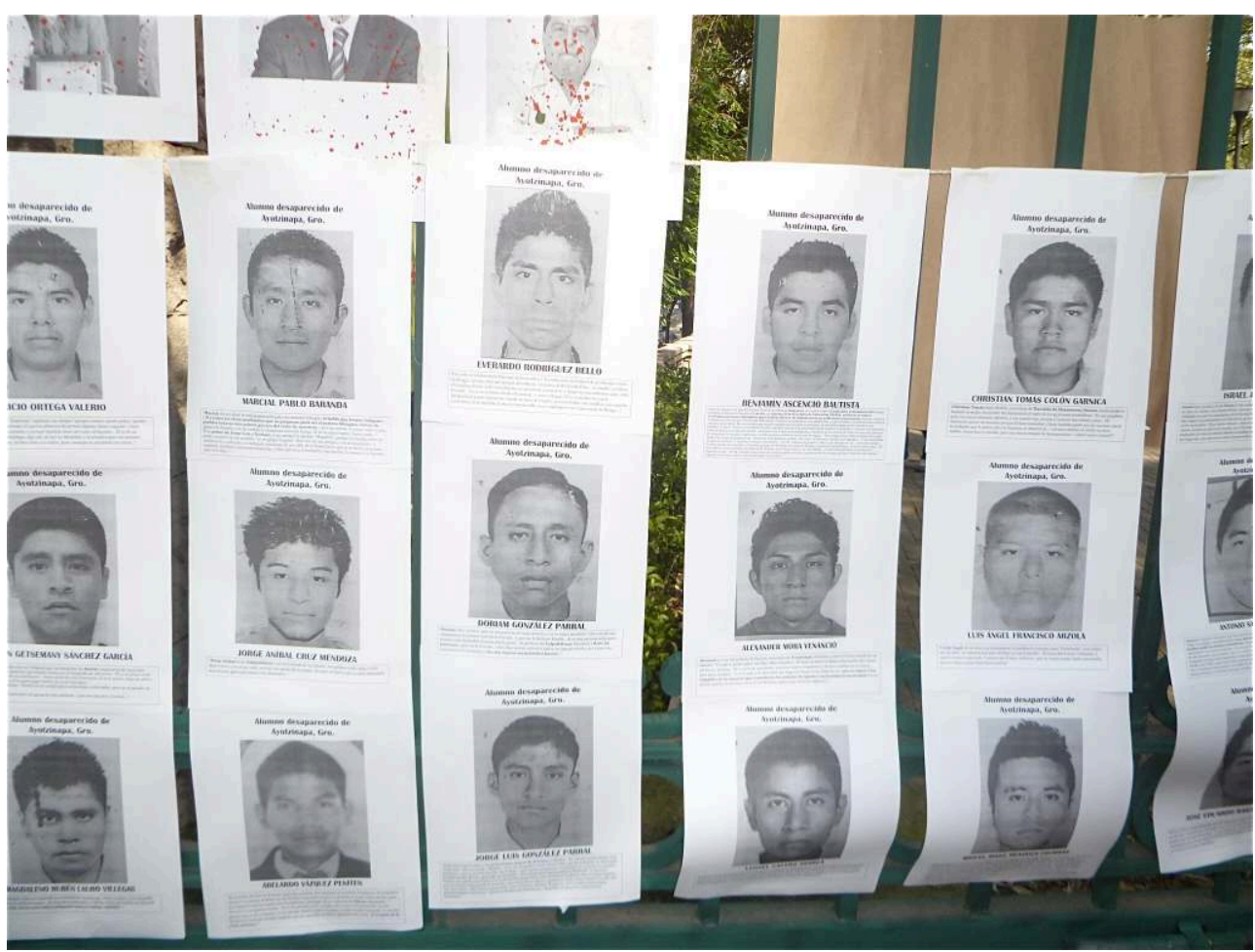

Même si la peinture des grands muralistes mexicains n'est plus d'époque, car trop idéologique, la tentation de la représentation persiste pour témoigner. Et puis, il y aura celle des monuments aux héros comme on les voit sur l'avenue Réforma. Et les murs de noms comme dans le Cimetière principal de Santiago. Mais ce qui est immédiat et absolument nécessaire, c'est un curetage général de la société selon la pratique des hijos argentins et de leurs charivaris. Et, plus tard, un mémorial comme le Musée de la mémoire et des droits de l'homme de Santiago et l'œuvre souterraine d'Alfredo Jaar (Géométrie de la conscience)... Mais peut-être que le plus grand obstacle au Mexique pour une conception véridique de la disparition forcée, c'est la puissance des relations symboliques liant les vivants aux morts, en particulier lors de la Fête des morts. Il sera bien difficile de ne pas transformer les disparus en desasparecidos. Mais la matrice de cette civilisation qui inverse la cosmétique occidentale ne se trouvait-elle pas à Teotihuacan : le Complejo muerte du Musée d'anthropologie de Mexico?

\section{BIBLIOGRAPHIE}

Amnesty International, rapport, 2013.

Barthes Roland, La Chambre claire. Note sur la photographie, Paris, Gallimard, 1980.

Bolaño Roberto, 2666, trad. fr. de Robert Amutio, Paris, Gallimard, 2011.

Daoud Omar, Devoir de mémoire, Alger, auto-édition, 2007. 
Déotte Jean-Louis, Brossat Alain, L'époque de la disparition, Paris, L’Harmattan, 2000.

Déotte Jean-Louis, Brossat Alain, La mort dissoute, Paris, L'Harmattan, 2005.

Déotte Jean-Louis, « Éléments d'esthétique disparitionniste », Revue Lignes, n 8, éd. Léo Scheer, mai 2002 .

Déotte Jean-Louis, «La vidéo à l'épreuve de la disparition (Julieta Hanono, Fiorenza Menini) », in Les images limites, M. Gaguebin et J. Milly (dir.), Paris, 2006.

Déotte Jean-Louis, «Video y desaparición », El resplandor. Revista de la Escuela de cine, Universidad de Valparaíso, 2015.

Déotte Jean-Louis, Cosmétiques. Simondon, Panofsky, Lyotard, à paraître, coll. MSH Paris Nord en ligne, 2015.

Foucault Michel, Histoire de la folie à l'âge classique, Paris, Gallimard, 1972.

Foucault Michel, Cours du Collège de France, 1976.

Gruzinski Serge, La colonisation de l'imaginaire. Sociétés indigènes et occidentalisation dans le Mexique espagnol, XVI-XVIII e siècle, Paris, Gallimard, 1988.

Gruzinski Serge, Histoire du Nouveau Monde (avec Carmen Bernand) : T.2 ; Les Métissages, Paris, Fayard, 1993.

Gruzinski Serge, Histoire de Mexico, Paris, Fayard, 1996.

Gruzinski Serge, L'Aigle et le Dragon. Démesure européenne et mondialisation au XVI ${ }^{e}$ siècle, Paris, Fayard, 2012.

Lefeuvre-Déotte Martine, «La politique des Mères », L'époque de la disparition. Politique et esthétique, A. Brossat et J.-L. Déotte (dir.), Paris, L'Harmattan, 2000.

Lefeuvre-Déotte Martine, « Desaparicion y ausencia de duelo ", Politicas y Estéticas de la Memoria, Nelly Richard (dir.), Chile, Editorial Cuarto Propio, 2000.

Lefeuvre-Déotte Martine, "Octobre 61, un crime sans transmission », revue Transeuropéennes, « Relier la ville », $\mathrm{n}^{\circ}$ 21, automne 2001 ; article également publié dans Hommes et Migrations, octobre 2001.

Lefeuvre-Déotte Martine, «Le peuple des bidonvilles sur les boulevards », Le 17 octobre, un crime d'État à Paris, Olivier Le Cour Grandmaison (dir.), La Dispute, 2001.

Lefeuvre-Déotte Martine, « L'effacement des traces, la mère, le politique », Revue SocioAnthropologie, « Traces », université de Paris X, $\mathrm{n}^{\circ} 12,2^{\mathrm{e}}$ semestre, 2002.

Lefeuvre-Déotte Martine, « Entretien avec Laura Bonaparte (mère de la Place de Mai), ligne fondatrice ", revue Drôle d'Époque, « Quel présent pour l'Argentine? », nº 11, automne 2002.

Lefeuvre-Déotte Martine, « La mort dissoute. Un cas : l'Argentine », revue Quasimodo, n 9, « Corps en guerre ", Montpellier, 2005.

Lefeuvre-Déotte Martine, «Entre vie et mort : le travail de Rithy Panh », revue Frontières, Université du Québec à Montréal, La disparition et la mort, Alice Verstraeten (dir.), à paraître, 2015. Lyotard Jean-François, La Guerre des Algériens, Paris, Galilée, 1989.

Schiller Friedrich, Lettres sur l'éducation esthétique de l'humanité, 1799.

Simondon Gilbert, L'individuation psychique et collective, Paris, Aubier, 1989. 
Simondon Gilbert, L'individuation à la lumière des notions de forme et d'information, Paris, Jérôme Million, 2005.

Swain Gladys, Gauchet Marcel, Dialogue avec l'insensé. Une autre histoire de la folie, Paris, Gallimard, 1994.

Tocqueville Alexis de, La démocratie en Amérique, Paris, Le Livre de Poche, t. 1, 1986.

Communications et conférences à des colloques :

Lefeuvre-Déotte Martine, «Les "Français" et la disparition politique : le déni », Valparaiso, août 2013 ,

Lefeuvre-Déotte Martine, «Les “folles” de la Place de Mai : l'incessant témoignage », « Femmes dans la violence de l'histoire. Le témoignage au féminin », colloque interdisciplinaire et international en partenariat entre l'université Paris Diderot-Paris 7 et le Mémorial de la Shoah, organisation : Any Dayan Rosenmann, 28 et 29 novembre 2010.

Valorisation de la recherche

France Culture, productrice et réalisatrice d'un documentaire « L'ombre de la disparition » pour la série Le monde en soi, 1h50, première diffusion le 3 novembre 2001.

\section{NOTES}

3. Si l'on compare les chiffres, la dictature argentine n'aura pas été plus « efficace » que l'état de dictature «molle» mise en place par le PRI (Parti révolutionnaire institutionnel), suivi du PAN, en leur temps. Le PRI avait mis en place un système de clientélisme inclusif, que ce soit pour obtenir un terrain à bâtir (" parachutisme»), un emploi dans une entreprise publique, etc. Sans compter la mainmise sur la culture, par exemple l'institution du fameux Musée d'anthropologie de Mexico, etc. Le relais des intellectuels et des artistes serait à étudier dans cette perspective.

4. Serge Gruzinski, Histoire de Mexico, Paris, Fayard, 1996. D'après d'autres informations, les normaliens d'Ayotzinapa voulaient se rendre à Mexico pour participer aux commémorations du massacre du 2 octobre, place des Trois Cultures.

5. Roberto Bolaño, 2666, trad. fr. de Robert Amutio, Paris, Gallimard, 2011.

6. Michel Foucault, Cours du Collège de France, 1976.

7. Serge Gruzinski, Histoire du Nouveau Monde (avec Carmen Bernand), t. 2, Les Métissages, Paris, Fayard, 1993. Et : La colonisation de l'imaginaire. Sociétés indigènes et occidentalisation dans le Mexique espagnol, XVI-XVIII siècle, Paris, Gallimard, 1988. La fantasmagorie du métissage n'est pas comme l'écrit notre auteur, un progrès par rapport à une anthropologie du " pur et de l'impur ». Elle a l'inconvénient à nos yeux de faire disparaître la pensée de la différence et $a$ fortiori une réflexion comme celle de Lyotard sur les «différends ». À qui profite l'apport des cultures autochtones dominées? Quand les artisans indiens comme à Tonantzintla et Acatepec, aux environs de Puebla, donnèrent des traits indiens aux angelots, les coiffant de panaches de plumes et en sculptant des guirlandes de fruits tropicaux et des épis de maïs, c'était bien à la gloire de la Vierge Marie imposée par les Espagnols! L'esthétique du «baroque » vient légitimer cette absorption du dominé par le dominant.

8. Serge Gruzinski, Histoire de Mexico, op. cit., 1996.

9. Cette affiche anti-discriminatoire émane d'une loi votée par le Parlement de Mexico District Féféral, plus à «gauche » que les autres parlements étatiques. Elle reconfigure l'appartenance symbolique à l'humanité.

10. Gladys Swain et Marcel Gauchet, Dialogue avec l'insensé. Une autre histoire de la folie, Paris, Gallimard, 1994. 
11. Michel Foucault, Histoire de la folie à l'âge classique, Paris, Gallimard, 1972.

12. Rapport d'Amnesty International de 2013.

13. Gilbert Simondon, L'individuation à la lumière des notions de forme et d'information, Paris, Jérôme Million, 2005.

14. Omar Daoud, Devoir de mémoire, Alger, auto-édition, 2007.

15. Serge Gruzinski, L'Aigle et le Dragon. Démesure européenne et mondialisation au XVI siècle, Paris, Fayard, 2012.

16. Alexis de Tocqueville, La démocratie en Amérique, Paris, Le Livre de Poche, t. 1, 1986.

17. Friedrich Schiller, Lettres sur l'éducation esthétique de l'humanité, 1799.

18. Notre : Cosmétiques. Simondon, Panofsky, Lyotard, à paraître, édition en ligne, 2015.

19. Jean-François Lyotard, La Guerre des Algériens, Paris, Galilée, 1989.

20. Gilbert Simondon, L'individuation psychique et collective, Paris, Aubier, 1989.

1. Les travaux de Martine Lefeuvre-Déotte cités en bibliographie.

2. Roland Barthes, La Chambre claire. Note sur la photographie, Paris, Gallimard, 1980.

\section{RÉSUMÉS}

Les normaliens d'Ayotzinapa dans l'État mexicain du Guerrero qui venaient protester à Iguala contre les liens de la mairie avec les narcos ont été attaqués par la police locale. Quarante-trois d'entre eux sont toujours portés disparus depuis le 26 septembre. L'État fédéral se contente des aveux de deux criminels: après "livraison» à leur gang, les jeunes futurs instituteurs, appartenant aux classes les plus pauvres, la plupart d'origine indienne, auraient été assassinés, leurs corps auraient été brûlés et leurs restes jetés dans la rivière locale. Un vaste mouvement de protestation secoue le pays, des édifices publics sont attaqués, les parents réclamant, comme en Argentine les Mères de la Place de Mai, « la réapparition en vie de leurs enfants $»^{1}$.

Or comme partout ailleurs, la photographie des disparus devient une arme de combat puisque c'est la seule preuve qu'ils ont été. S'ouvre alors une autre époque de l'esthétique dont Barthes ${ }^{2}$ est le premier théoricien.

\section{INDEX}

Mots-clés : disparitions forcées, photographie, individuation, esthétique de la disparition Index géographique : Mexique 